\title{
Paradigm shift in acute dizziness: is caloric testing obsolete?
}

\author{
Miranda Morrison ${ }^{1} \cdot$ Athanasia Korda $^{1} \cdot$ Ewa Zamaro $^{1} \cdot$ Franca Wagner $^{2} \cdot$ Marco D. Caversaccio $^{1} \cdot$ Thomas C. Sauter $^{3}$. \\ Roger Kalla ${ }^{4}$. Georgios Mantokoudis ${ }^{1}$ (i)
}

Received: 2 May 2021 / Revised: 11 June 2021 / Accepted: 14 June 2021

(c) The Author(s) 2021

\begin{abstract}
Objective Cold and warm water ear irrigation, also known as bithermal caloric testing, has been considered for over 100 years the 'Gold Standard' for the detection of peripheral vestibular hypofunction. Its discovery was awarded a Nobel Prize. We aimed to investigate the diagnostic accuracy of Caloric Testing when compared to the video head impulse test (vHIT) in differentiating between vestibular neuritis and vestibular strokes in acute dizziness.

Design Prospective cross-sectional study (convenience sample).

Setting All patients presenting with signs of an acute vestibular syndrome at the emergency department of a tertiary referral center.

Participants One thousand, six hundred seventy-seven patients were screened between February 2015 and May 2020 for Acute Vestibular Syndrome (AVS), of which 152 met the inclusion criteria and were enrolled. Inclusion criteria consisted of a state of continuous dizziness, associated with nausea or vomiting, head-motion intolerance, new gait or balance disturbance and nystagmus. Patients were excluded if they were younger than 18 years, if symptoms lasted $<24 \mathrm{~h}$ or if the index ED visit was $>72 \mathrm{~h}$ after symptom onset. Of the 152 included patients 85 completed testing. We assessed 58 vestibular neuritis and 27 stroke patients.

Main outcome measures All patients underwent calorics and vHIT followed by a delayed MRI which served as a gold standard for vestibular stroke confirmation.

Results The overall sensitivity and specificity for detecting stroke with a caloric asymmetry cut-off of $30.9 \%$ was $75 \%$ and $86.8 \%$, respectively [negative likelihood ratio (NLR) 0.29] compared to $91.7 \%$ and $88.7 \%$ for vHIT (NLR 0.094). Best VOR gain cut-off was 0.685 . Twenty-five percent of vestibular strokes were misclassified by calorics, $8 \%$ by vHIT.

Conclusions Caloric testing proved to be less accurate than vHIT in discriminating stroke from vestibular neuritis in acute dizziness. Contrary to classic teaching, asymmetric caloric responses can also occur with vestibular strokes and might put the patient at risk for misdiagnosis. We, therefore, recommend to abandon caloric testing in current practice and to replace it with vHIT in the acute setting. Caloric testing has still its place as a diagnostic tool in an outpatient setting.
\end{abstract}

Keywords Caloric testing $\cdot$ Head-impulse test $\cdot$ Neuritis $\cdot$ Acute stroke $\cdot$ Vertigo $\cdot$ Dizziness

Georgios Mantokoudis

georgios.mantokoudis@insel.ch

1 Department of Otorhinolaryngology, Head and Neck Surgery, Inselspital, University Hospital Bern and University of Bern, 3010 Bern, Switzerland

2 University Institute of Diagnostic and Interventional Neuroradiology, Inselspital, University Hospital Bern and University of Bern, Bern, Switzerland

3 Department of Emergency Medicine, Inselspital, University Hospital Bern and University of Bern, Bern, Switzerland

4 Department of Neurology, Inselspital, University Hospital Bern and University of Bern, Bern, Switzerland

\section{Introduction}

Since its discovery in 1907 by Robert Bárány [1], who received the Nobel prize in 1916 [2], Caloric Testing has been widely accepted as a Gold Standard for detecting a vestibular hypofunction, thus allowing the confirmation of peripheral vestibular disease in patients suffering from acute dizziness. However, the accuracy of calorics in discriminating vestibular neuritis from vestibular strokes in patients with acute dizziness is not known. One study reported a false negative rate of up to $22 \%$ of vestibular strokes [3]. Between 5 and $25 \%$ of isolated dizziness end up 
with a final diagnosis of posterior fossa infarction [3-5] with a reported initial misdiagnosis rate of up to $28 \%$ [6]. This is because central vestibular disorders mimic in many cases peripheral disease[7] also known as pseudo-neuritis. Fifty percent of dizzy patients with a stroke do not demonstrate any focal neurological signs [8]. Thermal (caloric) water irrigation of both ears stimulate the vestibular organ, particularly the horizontal semicircular canal, elicitating eye movements (nystagmus), which can be recorded with video Frenzels. This test, however, is very uncomfortable, consumes vast emergency department (ED) resources and is potentially less accurate due to great inter-subject and test-retest variability[9]. In view of all these disadvantages, any solution to avoid undertaking calorics and to increase diagnostic accuracy is crucial. Developed by Halmagyi and Curthoys [10], the Head Impulse Test (HIT) has proved itself as one of the most accurate triage tests in detecting vestibular strokes [3, 11]: Physicians have to move the patient's head from side to side rapidly (impulse) inducing the vestibulo-ocular reflex (VOR). Head and eye movements can be recorded quantitatively with VOG goggles (video-HIT, or vHIT) [12, 13]. Disconcordant eye and head movements (pathologic VOR) indicate a peripheral vestibular deficit, such as vestibular neuritis. An intact VOR (concordant eye/head movements) would, however, be indicative of vestibular stroke. With the advent of digital technologies such as eye- and head-tracking by video-oculography (VOG) [11], it has been possible to offer non-invasive, time- and cost-efficient diagnostic techniques in the ED. Although many studies have investigated the correlation between caloric testing and vHIT, none have focused on acute vestibular disorders [14, 15]. We hyphothesized that a pathologic caloric exam without any additional test such as the vHIT might give ED physicians a false sense of security and put stroke patients at risk being misdiagnosed as vestibular neuritis. Unfortunately, only a small proportion of physicians perform systematically HITs in the ED [16].

In our study, we aim to investigate the diagnostic accuracy of Caloric Testing when compared to vHIT in differentiating between vestibular neuritis and vestibular strokes in acute dizziness.

\section{Materials and methods}

We conducted a prospective cross-sectional study (convenience sample), between February 2015 and May 2020, of all cases presenting with acute dizziness at the ED in a tertiary referral center. 1677 patients were screened for Acute Vestibular Syndrome (AVS) as part of a large cross-sectional study (DETECT_-[Dizziness Evaluation Tool for Emergent Clinical Triage]), of which 152 met the inclusion criteria and were enrolled. Inclusion criteria consisted of a state of continuous dizziness, associated with nausea or vomiting, head-motion intolerance, new gait or balance disturbance and nystagmus. Patients were excluded if they were younger than 18 years, if symptoms lasted $<24 \mathrm{~h}$ or if the index ED visit was $>72 \mathrm{~h}$ after symptom onset. Figure 1S (Appendix) shows a flow diagram with all screened patients, inclusions and exclusions of dizzy patients. All enrolled patients underwent when feasible a thorough physical examination, Caloric Testing and vHIT testing. All patients received an MRI either at the index visit or a second, delayed MRI if there was no acute MRI indicated based on clinical grounds or if the first MRI was non-diagnostic. The delayed MRI served as a reference standard for stroke detection. Enrolled patients were clinically re-evaluated between day 3 and day 10 , at day 30 and day 90 . All images were reviewed by a certified second blinded neuroradiologist, discrepancies were resolved by consensus and inter-rater concordance reported. Figure 1 shows the two investigated tests, the required equipment, stimulation modalities and recording setup.

We performed caloric tests irrigating sequentially both ears with warm $\left(44^{\circ} \mathrm{C}\right)$ and cold $\left(30^{\circ} \mathrm{C}\right)$ water for $30 \mathrm{~s}$ and a total water volume of $250 \mathrm{ml}$ (Vario Otopront device) in patients lying $30^{\circ}$ supine (Fig. $2 \mathrm{~S}$, supplementum, panel A). Intervals between irrigations were 5 min long, starting first with warm irrigation on the right ear. Convection flows of inner ear fluid (particularly in the horizontal semicircular canal) produced horizontal eye movement responses (nystagmus), which were recorded in darkness (blocked visual fixation) with a calibrated VOG device (EyeSeeCam, Munich). The Cut-off for pathologic Caloric responses was $20 \%$ asymmetry [17], which was calculated using Jongkee's formula [18] after correcting for spontaneous nystagmus.

In contrast, vHIT was performed solely on the lateral canal by fast passive horizontal head movements (high frequency, $10-20^{\circ}$ head excursion in 100-300 ms corresponding to a $1000-6000^{\circ} / \mathrm{sec}^{2}$ acceleration) in room light during visual target fixation at $>1 \mathrm{~m}$ distance. We recorded head and eye movement velocity with a head mounted infrared highspeed camera (EyeSeeCam, Munich) connected to a laptop by USB (Fig. 2S, Panel B). VOR gain values were derived from eye velocity divided by head velocity at $60 \mathrm{~ms}$ after HIT onset [19]. vHIT exams were classified as abnormal based on VOR gains (Gain $<0.79$ based on own laboratory normative values) and the presence of corrective saccades. Additionally we collected information on age, gender, duration of symptoms, and other associated relevant otological or neurological symptoms.

\section{Statistics}

Cohen's Kappa was calculated for the assessment of interrater agreement between two experienced neuroradiologist. Descriptive statistics were reported using SPSS statistical 

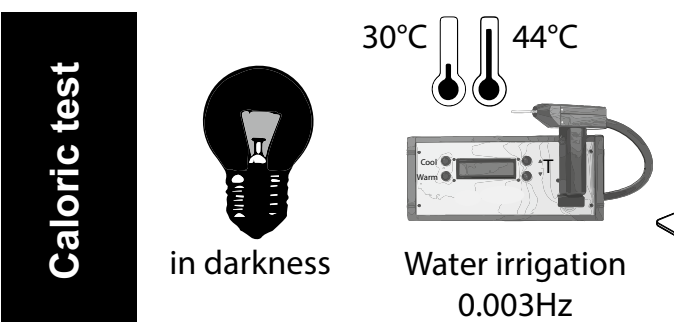

Eye Tracking

in darkness

Water irrigation $0.003 \mathrm{~Hz}$
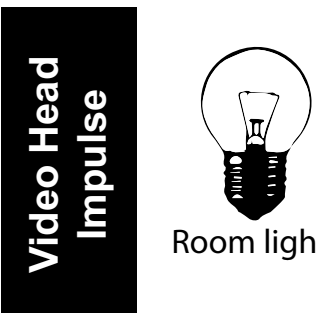

Head motion

$2-5 \mathrm{~Hz}$

Room light

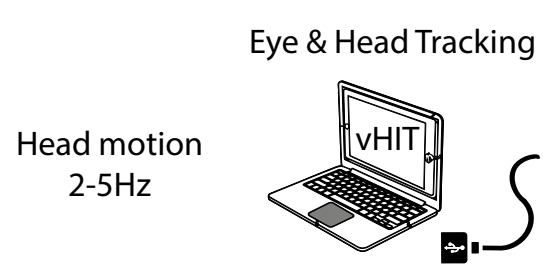

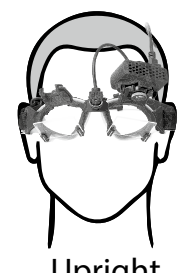

Upright

Recording

Position

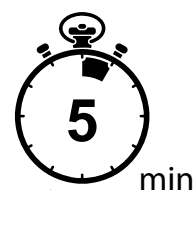

Duration
Fig. 1 Technical setup for the caloric exam compared to the VideoHead Impulse test. Diagram comparing the technical setup for the caloric exam with that of the vHIT; calorics are performed in the dark on a patient in a supine position and head rest positioned at $30^{\circ}$ from horizontal. The outer ear canal on each side is irrigated sequentially for $30 \mathrm{~s}$ (at $30^{\circ} \mathrm{C}$ cold and $44^{\circ} \mathrm{C}$ warm water) and the resulting eye movements recorded for a duration of 3 min using VOG goggles. The whole procedures takes up to 30 min including waiting intervals of 5 min between irrigations. The vHIT is performed in a normal lit room on a upright sitting patient. The head is moved rapidly from side to side (20 times in an impulse-like motion) and eye movements are recorded using adapted vHIT-goggles. When done correctly, the vHIT takes under 5 min software (IBM SPSS Statistics for Windows, Version 25.0. Armonk, NY: IBM Corp.). We used a binary logistic regression to evaluate stroke predictors derived from caloric and vHIT exams in 65 patients who underwent both test modalities. We calculated a receiver characteristics curve (ROC) with its corresponding sensitivity, specificity, accuracy and negative likelihood ratio with its impact on post-test probability for each test. Best cut-off points based on Youden's J. We followed the STARD guidelines for reporting the diagnostic accuracy. Our estimated sample size was 52 with an estimated marginal error of 0.1 (95\% CI $80 \%$ power) and a diagnostic accuracy (AUC) of 0.90 for vHIT. The two ROC curves were compared using the method of DeLong et al. [20].

\section{Results}

We screened 1677 patients with AVS of which 152 patients were enrolled aged between 20 and 91 (mean 55.67 years). Out of 152 patients, 58 were diagnosed with vestibular neuritis (mean age 54 years \pm 15.7 ), while the remaining 27 patients were vestibular strokes (mean age 62.1 years \pm 15.9 years). Vascular territories included the PICA (17), SCA (3), AICA (2), basilar artery (3), vertebral artery (2), anterior (1) and middle cerebral artery (4). There was an excellent inter-rater agreement regarding masked
MRI assessment $(94 \%, \kappa=0.78)$. None of the patients with a normal caloric response had an abnormal vHIT (Table 1). Patients with an abnormal vHIT, however, systematically showed a pathologic caloric response as well. Table 1 shows the number of concordant or disconcordant exams comparing vHIT with calorics. Every increase of 0.1 VOR gain increased significantly the stroke risk (OR 2.832, 95\% CI $1.5-5.2, P<0.001$, Table 2$)$. A decreased asymmetry of $1 \%$ steps, however, decreased slightly the stroke risk (OR 0.926, 95\% CI 0.88-0.97, $P=0.001$, Table 2). Figure 2 shows the receiver operating characteristic (ROC) curves for vHIT (AUC $=0.93,95 \%$ CI $0.84-1.00, P<0.001)$ and calorics (AUC $=0.86,95 \%$ CI $0.74-0.99, P<0.001$ ) with curves going to the left upper corner. There was no statistical difference between the two ROC curves $(P=0.22)$.

The overall sensitivity in discriminating strokes with caloric testing was $75 \%$ with a specificity of $86.8 \%$ (Table 3). The accuracy of caloric testing was $84.6 \%$ using a cut-off of $30.9 \%$ asymmetry. The accuracy of vHIT in detecting stroke was $89.3 \%$ with a sensitivity of $91.7 \%$ and specificity of $88.7 \%$ using a cut-off of 0.685 VOR gain. Table 3 shows alternative cut-off values and their corresponding sensitivity/specificity. The negative likelihood ratio for ruling-out stroke was 0.288 for calorics and 0.094 for vHIT (Table 3). Table 4 shows the pre-test and posttest probabilities of stroke assuming pre-test probabilities based on risk stratification rules. Table 4 illustrates the 
Table 1 Concordance vHIT versus calorics

\begin{tabular}{|c|c|c|c|c|c|}
\hline \multirow{2}{*}{\multicolumn{2}{|c|}{ Vestibular function }} & \multicolumn{3}{|c|}{$\begin{array}{c}\text { Video Head Impulse Test } \\
\text { (vHIT) }\end{array}$} & \multirow[b]{2}{*}{ Total } \\
\hline & & Normal & $\begin{array}{c}\text { Hypo- } \\
\text { function } \\
\text { right }\end{array}$ & $\begin{array}{c}\text { Hypo- } \\
\text { function } \\
\text { left }\end{array}$ & \\
\hline \multirow{4}{*}{$\begin{array}{l}\text { Bithermal } \\
\text { Caloric Test } \\
\text { (calorics) }\end{array}$} & Normal & 5 & 0 & 0 & 5 \\
\hline & Hypofunction right & 5 & 20 & 0 & 25 \\
\hline & Hypofunction left & 5 & 0 & 25 & 30 \\
\hline & Bilateral Hypofunction & 2 & 0 & 0 & 2 \\
\hline \multicolumn{2}{|l|}{ Total } & 17 & 20 & 25 & 62 \\
\hline
\end{tabular}

Table 2 Stroke risk estimation for vHIT and caloric asymmetry

\begin{tabular}{|c|c|c|c|c|c|c|c|c|c|}
\hline & \multirow[t]{2}{*}{$\begin{array}{l}\text { Increment } \\
\text { steps }\end{array}$} & \multirow[t]{2}{*}{$\begin{array}{l}\text { Regression } \\
\text { coefficient }\end{array}$} & \multirow[t]{2}{*}{ Standard error } & \multirow[t]{2}{*}{ Wald } & \multirow[t]{2}{*}{$d f$} & \multirow[t]{2}{*}{$P$ Value } & \multirow[t]{2}{*}{ Odd's ratio } & \multicolumn{2}{|c|}{$\begin{array}{l}95 \% \text { Confidence } \\
\text { interval }\end{array}$} \\
\hline & & & & & & & & Lower & Upper \\
\hline vHIT gain & 0.1 & 1.041 & 0.308 & 11.387 & 1 & 0.001 & 2.832 & 1.547 & 5.183 \\
\hline Caloric asymmetry & $1 \%$ & -0.077 & 0.024 & 10.446 & 1 & 0.001 & 0.926 & 0.883 & 0.970 \\
\hline
\end{tabular}

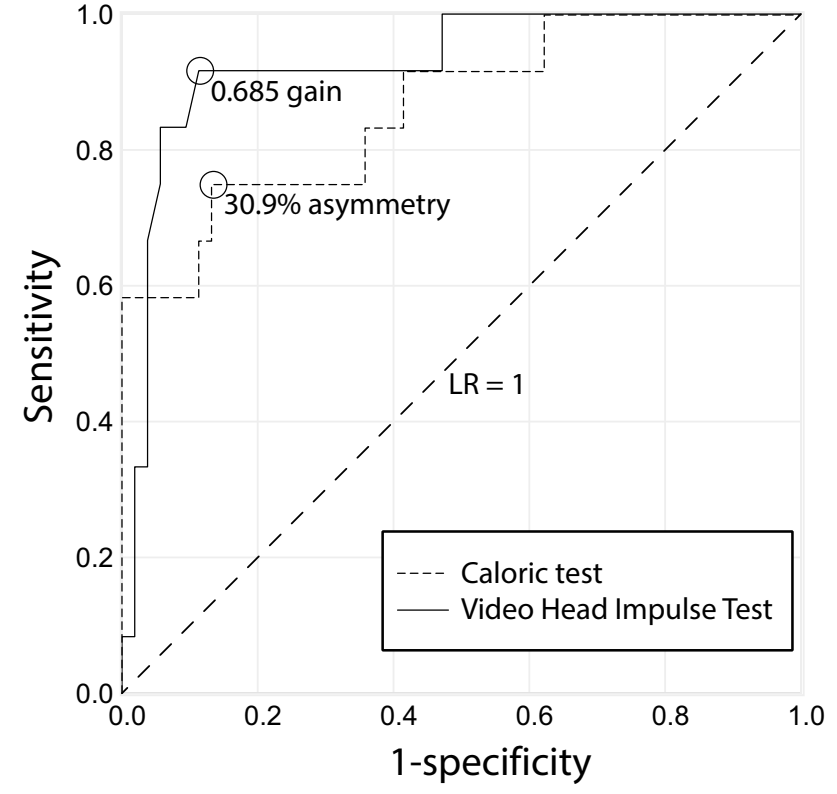

Fig. 2 ROC curves. ROC curve demonstrating a higher sensitivity and specificity for vHIT for the detection of stroke compared to calorics. Black circles indicate the optimal test discrimination cut-off for each test. The dotted line illustrates a likelihood ratio of 1 with an area under the curve (AUC) at 0.5 indicating an unhelpful test

impact of the negative likelihood ratio (NLR) on stroke probability. Stroke probability decreased by $9-53 \%$ points after a vHIT exam and by $6.9-28.6 \%$ points after calorics depending on the assumed pre-test probability.

\section{Discussion}

The presence of caloric asymmetry is used in the emergency department as a means of confirming neuritis in patients with AVS and complete absence of neurological symptoms. If an asymmetrical caloric test was synonymous of pathological peripheral function (neuritis), every fourth vestibular stroke would have been missed. We confirmed our hypothesis that a pathological caloric test without additional clinical tests such as the bedside vHIT puts a stroke patient at risk being misclassified as a vestibular neuritis. Considering currently accepted test cut-off values for caloric response asymmetry, even every $2^{\text {nd }}$ to $3^{\text {rd }}$ patient would have been misdiagnosed. On the contrary, a vHIT test yields a significantly higher sensitivity and specificity for vestibular stroke detection. An increased gain value was significantly associated with stroke. A pathological vHIT result with low gain was, however, systematically associated with a pathological Caloric Test result. We never observed a normal caloric test when vHIT was abnormal and thus, there is no need to perform any caloric testing to confirm a pathologic vHIT.

\section{Is caloric testing accurate for discrimination between peripheral and central dizziness?}

Admittedly, for calorics, we observed a decent decrease of stroke risk for every $1 \%$ of increase in asymmetry, which 
Table 3 Sensitivity and specificity for vHIT and calorics

\begin{tabular}{lllll}
\hline & \multicolumn{2}{l}{ vHIT(Gain) } & \multicolumn{2}{l}{ Calorics (\% Asymmetry) } \\
\hline Test cut-off & $>0.685$ & $>0.805$ & $<25.3 \%$ & $<30.9 \%$ \\
AUC (95\% CI) & $0.926(0.833-0.976)$ & $0.863(0.755-0.936)$ & \\
Sensitivity & $91.7 \%$ & $41.7 \%$ & $58.3 \%$ & $75 \%$ \\
Specificity & $88.7 \%$ & $96.2 \%$ & $96.2 \%$ & $86.8 \%$ \\
Negative test & 48 & 58 & 56 & 49 \\
Positive test & 17 & 7 & 9 & 16 \\
True positives & 11 & 5 & 7 & 9 \\
False positives & 6 & 2 & 2 & 7 \\
True negatives & 47 & 51 & 51 & 46 \\
False negatives & 1 & 7 & 5 & 3 \\
Likelihood ratio pos. test & 8.097 & 11.042 & 15.458 & 5.679 \\
Likelihood ratio neg. test & 0.094 & 0.606 & 0.433 & 0.288 \\
Accuracy & 89.3 & 86.2 & 89.2 & 84.6 \\
\hline
\end{tabular}

Table 4 Pre-test and post-test probabilities of stroke using calorics or vHIT to 'rule out' stroke

\begin{tabular}{lll}
\hline \multicolumn{2}{l}{ Post-test probability of stroke } \\
\hline Test & Calorics (rule out stroke) & vHIT (rule out stroke) \\
Test cut-off & $30.9 \%$ asymmetry & 0.685 gain \\
Pre-test probability of stroke (based on risk & Sn 75\%, Sp 86.8\% & Sn 91.7\%, Sp 88.7\% \\
stratification rules) & NLR 0.29 & NLR 0.094 \\
$10 \%$ (low) & $3.1 \%$ & $1.0 \%$ \\
$25 \%$ (average) & $8.8 \%$ & $3.0 \%$ \\
$50 \%$ (high) & $22.4 \%$ & $8.6 \%$ \\
$75 \%$ (very high) & $46.4 \%$ & $22.0 \%$ \\
\hline
\end{tabular}

$S n$ sensitivity, $S P$ specificity, $N L R$ negative likelihood ratio was not described in the literature before. The cut-off value, however, to rule-out stroke was higher (31\% asymmetry) compared to the test cut-off used in laboratories (20-25\%) for the detection of vestibular deficits [17]. We demonstrated, that central lesions can cause pathologic caloric responses in every 4th patient, which is in line with the current literature[3] (supplemental material). Thus, a pathologic caloric response is not a hallmark for a peripheral lesion but rather documents a deficit of vestibular pathways at any neuronal level in the low frequency range.

\section{vHIT is more sensitive for vestibular stroke detection}

Each incremental increase in VOR gain in vHIT resulted, however, in a significant incremental increase in stroke risk. Our test cut-off of 0.685 gain confirmed previously reported test thresholds for stroke discrimination with vHIT [21]. Based on current literature, we recommend, however, to assess additionally the gain asymmetry and the corrective saccade amplitude in order to further increase the accuracy of vHIT in detecting stroke [22]. Even clinically performed HITs (which assess VOR function qualitatively by simple eye observation looking for corrective saccades) yield a high sensitivity of stroke detection in acute dizziness, provided that they are performed by experts. However, a quantitative method, such as vHIT, would allow for more reliable and more examiner independent results. A recent study from our group showed, that even non-experts and novices were able to perform valid HITs using a video recording system [23]. Thus, a point-of care examination with vHIT in the ED has the potential of a widespread use, providing an accurate, cost-efficient and non-invasive method for stroke detection in acute dizzy patients.

\section{Can the caloric test be replaced by the vHIT in the acute setting?}

vHIT demonstrated a better accuracy with a higher sensitivity/specificity in the detection of vestibular strokes. The rate of missed patients (false negative result) having a serious cause of dizziness was significantly lower with vHIT. The false positive rate, however, was the same for both tests. In a previous cross-sectional study, vHIT was found to be even more sensitive for stroke detection than MRI [21, 24] [21], if performed within $24 \mathrm{~h}$ [3]. This finding was confirmed by 
others $[25,26]$. There are no publications investigating or comparing the accuracy of vHIT versus calorics in detecting vestibular strokes in patients with an acute vestibular syndrome. Both exams have been extensively compared regarding the detection of vestibular deficits in sub-acute or chronic stages [14, 15], however, such deficits might originate from peripheral or central causes. Rather than seeking to initially detect a benign vestibular deficit with calorics, emergency physicians and general practitioners should prioritise the exclusion first and foremost of any dangerous cause of acute dizziness with vHIT. Benign causes of dizziness could be further assessed and treated as a second line, for example in an outpatient, sub-acute setting. We therefore suggest a paradigm shift towards modern vHIT testing in patients with acute dizziness and to abandon calorics in the acute setting.

\section{When to perform calorics}

There are differences reported in the literature regarding the detection of vestibular deficits $[14,15]$, not specifically vestibular strokes. Comparing vHITs to calorics is like comparing apples to pears [27]; Calorics represent the measurement of low frequency stimulation of horizontal semicircular canals only, whereas vHITs concentrate of high frequencies and test all six canals in all spatial planes. It is, therefore, not surprising to find a dissociation of the two.

Other articles did compare the clinical HIT (before the advent of video-oculography) with calorics [28]. These articles support the idea that there is a low correlation between both exams, the vHIT often having a very low sensitivity for the detection of canal paresis $[14,15]$. A dissociation with abnormal calorics and normal vHIT can also be seen in patients with a mild vestibular hypofunction if this canal paresis exceeds $\sim 40 \%$ caloric asymmetry [28-30]. vHIT sensitivity in detecting acute vestibular deficits is higher (63\%) and lower in chronic dizziness (33\%) [31] with an overall reported sensitivity ranging from 41 to $86 \%$ [25]. Caloric tests, however, are more sensitive to diagnose Menière's disease [32] being often abnormal while vHIT is not affected. Calorics measure, however, only one single semicircular canal function and might miss an incomplete neuritis (inferior neuritis) [33].

Because the range calorics measure differ from vHIT (low frequency vs high frequency), it should not completely be excluded altogether. Rather, it should be seen as a complementary exam of vestibular function. Thus, patients presenting with a clinical picture of AVS, with a normal delayed MRI (3-10 days after symptom onset) and a normal vHIT, would be good candidates for further investigations by Calorics. If this is the case, one could argue for the redundancy of caloric testing in the acute setting and argue for its relegation to a later phase of non-acute testing to extend investigations of vestibular function. However, there is no need to perform calorics if vHIT is abnormal, since we never observed a dissociation of the two tests when vHIT was abnormal.

\section{Strength and limitations}

Our paper is the first large study offering a direct comparison of Caloric Testing and vHIT in acute vestibular syndrome, however, our results are not generalizable to all dizzy patients. In addition, we did not assess the absolute canal function or the sum of the peak slow phase velocity of caloric nystagmus, since absolute values would not be generalizable due to the absence of any normalization procedure. Reporting the asymmetry of canal function alone is, however, not meaningful in patients with a bilateral vestibulopathy or presbyvestibulopathy. In addition, there is large variability regarding the thermal energy applied to the lateral canals compared to standardized horizontal head movements in vHIT.

Careful vHIT interpretation is advised in patients with other pathologies such as e.g. Menière's Disease, vestibulare Schwannoma [25], vestibular migraine or BPPV, which can cause episodic dizziness and thus, bearing the risk of finding an asymptomatic patient at the examination time point. Best sensitivity of vHIT is yielded in patients having continuous dizziness associated with spontaneous nystagmus, which serves as an objective clinical sign of dizziness and underlying severity of vestibular imbalance. We also had a larger number of vHIT results versus caloric results; this could be due to a refusal by highly symptomatic patients to undergo caloric investigation. This could potentially lead to a selection bias in that highly symptomatic patients where inadvertently excluded from the study.

\section{Clinical implication}

Our study results have an immediate impact in current clinical practice suggesting a paradigm shift from calorics towards a modern vHIT exam. Caloric testing has a variety of limitations: (1) only one semicircular canal (horizontal) is stimulated per ear while the remaining four canals remain unassessed, (2) the stimulus is non-physiological stimulating at low frequencies only $(<0.003 \mathrm{~Hz})$ and non-reciprocal (stimulus from the contralateral ear missing), (3) it is very disagreeable for patients, inducing vertigo lasting up to several minutes, (4) there is a large inter-subject variability due to ear anatomy resulting in a variable application of thermal energy, (5) it needs a special and stationary irrigation device to maintain a constant water temperature with a purified water supply, (6) has to be performed in total darkness (adapted room) with Frenzel or video Frenzel goggles to remove visual fixation and finally, (7) it is costly consuming both, vast human and time resources. We, therefore, suggest 
to replace calorics with a more convenient and simple vHIT in AVS patients in view of its non-inferiority.

\section{Conclusions}

Caloric testing proved to be less accurate than vHIT in discriminating neuritis from vestibular stroke in acute dizziness. Contrary to classic teaching, asymmetric caloric responses can also occur with vestibular strokes and might put the patient at risk for misdiagnosis. We, therefore, recommend to abandon caloric testing in current practice. vHIT could serve as a replacement test in the acute setting. Caloric testing has still its place as a diagnostic tool in an outpatient setting.

\section{Data sharing statement}

The data that support the findings of this study are available from the corresponding author, [GM], upon reasonable request.

Supplementary Information The online version contains supplementary material available at https://doi.org/10.1007/s00415-021-10667-7.

Acknowledgements We would like to thank Mr. Gianni Pauciello, photographer, for the illustrations.

Author contributions MM: drafting the article, data analysis, critical revision of the article, final approval of the version to be published. AK: data collection, database setup, critical revision of the article, final approval of the version to be published. EZ: data collection, database setup, critical revision of the article, final approval of the version to be published. FW: data imaging analysis, critical revision of the article, final approval of the version to be published. MC: supervision, funding acquisition, critical revision of the article, final approval of the version to be published. TS: patient recruitment, critical revision of the article, final approval of the version to be published. RK: conceptualization, critical revision of the article, final approval of the version to be published. GM: conceptualization, methodology, data analysis, drafting the article, supervision, project administration, funding acquisition.

Funding Open Access funding provided by Universität Bern. This study was supported by the Swiss National Science Foundation \#320030_173081.

Availability of data and materials The lead author affirms that this manuscript is an honest, accurate, and transparent account of the study being reported; that no important aspects of the study have been omitted; and that any discrepancies from the study as planned (and, if relevant, registered) have been explained.

\section{Declarations}

Conflicts of interest None of the investigators has any relevant financial interests, activities, relationships, or affiliations that represent a relevant financial conflict of interest with respect to the conduct or analysis of this study. Eyeseetec company loaned the VOG goggles.

Dissemination to participants and related patient and public communities There are no plans to disseminate the results of the research to study participants or the relevant patient community.

Ethics All enrolled patients gave written consent. This study was approved by the local ethics committee (IRB).

Consent to participate Informed consent was obtained from all individual participants included in the study.

Consent for publication Not applicable.

Open Access This article is licensed under a Creative Commons Attribution 4.0 International License, which permits use, sharing, adaptation, distribution and reproduction in any medium or format, as long as you give appropriate credit to the original author(s) and the source, provide a link to the Creative Commons licence, and indicate if changes were made. The images or other third party material in this article are included in the article's Creative Commons licence, unless indicated otherwise in a credit line to the material. If material is not included in the article's Creative Commons licence and your intended use is not permitted by statutory regulation or exceeds the permitted use, you will need to obtain permission directly from the copyright holder. To view a copy of this licence, visit http://creativecommons.org/licenses/by/4.0/.

\section{References}

1. Barany R (1907) Physiologie und Pathologie (Funktions-Prüfung) des Bogengang-Apparates beim Menschen: Klinische Studien: F. Deuticke

2. Baloh RW (2002) Robert Barany and the controversy surrounding his discovery of the caloric reaction. Neurology 58:1094-1099

3. Newman-Toker DE, Kattah JC, Alvernia JE, Wang DZ (2008) Normal head impulse test differentiates acute cerebellar strokes from vestibular neuritis. Neurology 70:2378-2385

4. Casani AP, Dallan I, Cerchiai N, Lenzi R, Cosottini M, SellariFranceschini S (2013) Cerebellar infarctions mimicking acute peripheral vertigo: how to avoid misdiagnosis? Otolaryngol Head Neck Surg 148:475-481

5. Norrving B, Magnusson M, Holtas S (1995) Isolated acute vertigo in the elderly; vestibular or vascular disease? Acta Neurol Scand 91:43-48

6. Masuda Y, Tei H, Shimizu S, Uchiyama S (2013) Factors associated with the misdiagnosis of cerebellar infarction. J Stroke Cerebrovasc Dis 22:1125-1130

7. Braun EM, Tomazic PV, Ropposch T, Nemetz U, Lackner A, Walch C (2011) Misdiagnosis of acute peripheral vestibulopathy in central nervous ischemic infarction. Otol Neurotol 32:1518-1521

8. Kattah JC, Talkad AV, Wang DZ, Hsieh YH, Newman-Toker DE (2009) HINTS to diagnose stroke in the acute vestibular syndrome: three-step bedside oculomotor examination more sensitive than early MRI diffusion-weighted imaging. Stroke 40:3504-3510

9. Stockwell CW (1997) Vestibular testing: past, present, future. $\mathrm{Br}$ J Audiol 31:387-398

10. Halmagyi GM, Curthoys IS (1988) A clinical sign of canal paresis. Arch Neurol 45:737-739 
11. Newman-Toker DE, Curthoys IS, Halmagyi GM (2015) Diagnosing stroke in acute vertigo: the HINTS family of eye movement tests and the future of the "eye ECG." Semin Neurol 35:506-521

12. Newman-Toker DE, Saber Tehrani AS, Mantokoudis G, Pula JH, Guede CI, Kerber KA et al (2013) Quantitative video-oculography to help diagnose stroke in acute vertigo and dizziness: toward an ECG for the eyes. Stroke 44:1158-1161

13. Mantokoudis G, Tehrani AS, Wozniak A, Eibenberger K, Kattah JC, Guede CI et al (2015) VOR gain by head impulse videooculography differentiates acute vestibular neuritis from stroke. Otol Neurotol 36:457-465

14. Burston A, Mossman S, Mossman B, Weatherall M (2018) Comparison of the video head impulse test with the caloric test in patients with sub-acute and chronic vestibular disorders. J Clin Neurosci 47:294-298

15. Mezzalira R, Bittar RSM, do Carmo-Bilecki-Stipsky MM, Brugnera C, Grasel SS (2017) Sensitivity of caloric test and video head impulse as screening test for chronic vestibular complaints. Clinics (Sao Paulo) 72:469-473

16. Goeldlin M, Gaschen J, Kammer C, Comolli L, Bernasconi CA, Spiegel R et al (2019) Frequency, aetiology, and impact of vestibular symptoms in the emergency department: a neglected red flag. J Neurol 266:3076-3086

17. Wuyts F, Boniver R (2008) Normative data in ENG and VNG. B ENT 3:45-48

18. Furman JM, Jacob RG (1993) Jongkees' formula re-evaluated: order effects in the response to alternate binaural bithermal caloric stimulation using closed-loop irrigation. Acta Otolaryngol 113:3-10

19. Zamaro E, Saber Tehrani AS, Kattah JC, Eibenberger K, Guede CI, Armando L et al (2020) VOR gain calculation methods in video head impulse recordings. J Vestib Res 30:225-234

20. DeLong ER, DeLong DM, Clarke-Pearson DL (1988) Comparing the areas under two or more correlated receiver operating characteristic curves: a nonparametric approach. Biometrics 44:837-845

21. Mantokoudis G, Saber Tehrani AS, Wozniak A, Eibenberger K, Kattah JC, Guede CI et al (2015) VOR gain by head impulse video-oculography differentiates acute vestibular neuritis from stroke. Otol Neurotol 36:457-465
22. Chen L, Todd M, Halmagyi GM, Aw S (2014) Head impulse gain and saccade analysis in pontine-cerebellar stroke and vestibular neuritis. Neurology 83:1513-1522

23. Korda A, Sauter TC, Caversaccio MD, Mantokoudis G (2021) Quantifying a learning curve for video head impulse test: pitfalls and pearls. Front Neurol 11:615651. https://doi.org/10.3389/fneur. 2020.615651

24. Newman-Toker DE, Kerber KA, Hsieh YH, Pula JH, Omron R, Saber Tehrani AS et al (2013) HINTS outperforms ABCD2 to screen for stroke in acute continuous vertigo and dizziness. Acad Emerg Med 20:986-996

25. Alhabib SF, Saliba I (2017) Video head impulse test: a review of the literature. Eur Arch Otorhinolaryngol 274:1215-1222

26. Nelson JA, Viirre E (2009) The clinical differentiation of cerebellar infarction from common vertigo syndromes. West J Emerg Med 10:273-277

27. Jorns-Haderli M, Straumann D, Palla A (2007) Accuracy of the bedside head impulse test in detecting vestibular hypofunction. $\mathrm{J}$ Neurol Neurosurg Psychiatry 78:1113-1118

28. Perez N, Rama-Lopez J (2003) Head-impulse and caloric tests in patients with dizziness. Otol Neurotol 24:913-917

29. Bartolomeo M, Biboulet R, Pierre G, Mondain M, Uziel A, Venail F (2014) Value of the video head impulse test in assessing vestibular deficits following vestibular neuritis. Eur Arch Otorhinolaryngol 271:681-688

30. Bell SL, Barker F, Heselton H, MacKenzie E, Dewhurst D, Sanderson A (2015) A study of the relationship between the video head impulse test and air calorics. Eur Arch Otorhinolaryngol 272:1287-1294

31. Mahringer A, Rambold HA (2014) Caloric test and video-headimpulse: a study of vertigo/dizziness patients in a community hospital. Eur Arch Otorhinolaryngol 271:463-472

32. Hannigan IP, Welgampola MS, Watson SRD (2019) Dissociation of caloric and head impulse tests: a marker of Meniere's disease. J Neurol 268:431-439

33. Kim JS, Kim HJ (2012) Inferior vestibular neuritis. J Neurol 259:1553-1560 